\title{
POLYHEDRAL MODELS AND GEOMETRIC STRUCTURES FOR NANOTUBES
}

\author{
RICHARD K. F. LEE
}

(Received 25 January 2011)

\begin{abstract}
2010 Mathematics subject classification: primary 51P05; secondary 68U20, 82D80.
Keywords and phrases: polyhedral models, nanotubes, nanostructure, carbon nanotubes, silicon nanotubes, boron nanotubes, distinct bond lengths, distinct bond angles, molecular dynamics simulations.
\end{abstract}

In this thesis, some new polyhedral models for nanotubes are examined. The conventional rolled-up model for carbon nanotubes [4-6] assumes that a flat sheet of graphene is rolled into a seamless right circular cylinder and therefore in terms of the geometric parameters, the curvature inherent in the structure of nanotubes is not taken into account. The conventional rolled-up model of nanotubes completely ignores any effects due to curvature while the existing ideal polyhedral models for single-walled carbon nanotubes [1, 2] and boron nitride nanotubes [3], which are both hexagonal structures, are known to give predictions for the geometric parameters of the tube which are in excellent agreement with computational studies (molecular dynamics simulations and ab initio calculations). In this thesis the notion of an ideal polyhedral model is extended to silicon [7] and boron [8] nanotubes, which adopt respectively squares or skew rhombi and flat equilateral triangles as their structure. The silicon nanotubes considered here are assumed to be formed by $\mathrm{sp}^{3}$ hybridization and thus the nanotube lattice is assumed to comprise only squares or skew rhombi. The boron nanotubes considered here are assumed to be formed by complex bonding type and therefore the nanotube lattice is assumed to comprise a triangular pattern. From molecular dynamics simulation results for carbon nanotubes and silicon nanotubes, the bond lengths are known to vary depending upon the bond direction. Often this aspect cannot be ignored and therefore in this thesis both the conventional and the ideal polyhedral models are extended to include distinct bond lengths, and specifically for carbon, silicon and boron nanotubes. These general models are shown to be in excellent agreement with computational studies.

Thesis submitted to The University of Wollongong, June 2010. Degree approved, November 2010. Supervisors: Professor J. M. Hill and Dr B. J. Cox.

(C) 2011 Australian Mathematical Publishing Association Inc. 0004-9727/2011 \$16.00 
We first present the standard geometric parameters for the conventional nanotube model. Noting again that the curvature inherent in this model is completely ignored, for the ideal polyhedral model for silicon nanotubes we begin with three fundamental postulates, while for boron nanotubes we begin with two fundamental postulates. After some application of straightforward geometry, trigonometry and algebra, new formulae are derived which provide precise analytical expressions for geometric parameters such as radii, bond angles and thickness. Asymptotic expansions of these equations for quantities up to the first two orders of magnitude show that the first term gives the conventional model, while the second term may be viewed as a first-order correction to the conventional model. Geometric properties of ultrasmall nanotubes are examined which have certain extreme faceted structures.

Both the conventional and the ideal polyhedral models are then extended to include distinct bond lengths, including a general rolled-up model and a general polyhedral model. The general polyhedral model is similar to the ideal polyhedral model in that it is also based on certain well-defined postulates. For carbon nanotubes there are two general polyhedral models [10], termed Model I and II. Model I is a polyhedral model with distinct bond lengths and distinct bond angles. Model II is a polyhedral model with distinct bond lengths but with equal bond angles. In other words, the difference between the two carbon models is the assumed values of the bond angles. Model I assumes three prescribed distinct bond angles while Model II assumes that all the bond angles are the same. For both the silicon [11] and boron [9] nanotubes, there is only one general polyhedral model. From asymptotic expansions, the general polyhedral models include the rolled-up and ideal models or corresponding general cases, and therefore the general polyhedral model incorporates both rolled-up models and the ideal polyhedral model. Finally, the ideal polyhedral model and the general polyhedral model are compared with results obtained from molecular dynamics simulations and $a b$ initio calculations. The ideal polyhedral models appear to be in good agreement with simulation results, and the results for the general polyhedral models and other computational studies are also in excellent agreement [12].

In summary, the major original contribution contained in this thesis is a development of the existing ideal polyhedral models to encompass squares or skew rhombi and flat equilateral triangles for silicon and boron nanotubes respectively. We also provide new general polyhedral models for hexagonal, skew rhombi and flat equilateral triangular structures which incorporate distinct bond lengths.

\section{References}

[1] B. J. Cox and J. M. Hill, 'Exact and approximate geometric parameters for carbon nanotubes incorporating curvature', Carbon 45 (2007), 1453-1462.

[2] B. J. Cox and J. M. Hill, 'Geometric structure of ultra-small carbon nanotubes', Carbon 46 (2008), 711-713.

[3] B. J. Cox and J. M. Hill, 'Geometric model for boron nitride nanotubes incorporating curvature', J. Phys. Chem. C 112 (2008), 16 248-16 255.

[4] M. S. Dresselhaus, G. Dresselhaus and R. Saito, 'Carbon fibers based on C60 and their symmetry', Phys. Rev. B 45 (1992), 6234-6242. 
[5] M. S. Dresselhaus, G. Dresselhaus and R. Saito, 'Physics of carbon nanotubes', Carbon 33 (1995), 883-891.

[6] R. A. Jishi, M. S. Dresselhaus and G. Dresselhaus, 'Symmetry properties of chiral carbon nanotubes', Phys. Rev. B 47 (1993), 16 671-16 674.

[7] R. K. F. Lee, B. J. Cox and J. M. Hill, 'Idealized polyhedral model and geometric structure for silicon nanotubes', J. Phys.: Condens. Matter 21 (2009), 075301.

[8] R. K. F. Lee, B. J. Cox and J. M. Hill, 'Exact polyhedral model for boron nanotubes', J. Phys. A: Math. Theor. 42 (2009), 065204.

[9] R. K. F. Lee, B. J. Cox and J. M. Hill, 'Ideal polyhedral model for boron nanotubes with distinct bond lengths', J. Phys. Chem. C 113 (2009), 19 794-19 805.

[10] R. K. F. Lee, B. J. Cox and J. M. Hill, 'General rolled-up and polyhedral models for carbon nanotubes', Fullerenes, Nanotubes and Carbon Nanostructures (2010), accepted for publication.

[11] R. K. F. Lee, B. J. Cox and J. M. Hill, 'Silicon nanotubes with distinct bond lengths', J. Math. Chem. 47 (2010), 569-589.

[12] R. K. F. Lee, B. J. Cox and J. M. Hill, 'The geometric structure of single-walled nanotubes', Nanoscale 2 (2010), 859-872.

RICHARD K. F. LEE, Nanomechanics Group, School of Mathematical Sciences, The University of Adelaide, Adelaide, SA 5005, Australia

e-mail: richard.lee@adelaide.edu.au 